\title{
Does Clear Corneal Cataract Surgery Influence Conjunctivochalasis?
}

\author{
Tatsuya Mimura 1,2,3 MD, PhD; Michiko lida ${ }^{2}$, MD; Hidetaka Noma ${ }^{4}$, MD, PhD; Yuko Kamei ${ }^{2}$, MD \\ Aki Kondo ${ }^{2,3}$, MD, PhD; Maiko Yoshida ${ }^{2,3}$, MD; Manami Oguri ${ }^{2}$, MD; Yuka Tanaka ${ }^{2}$, MD \\ Atsushi Mizota ${ }^{1}, \mathrm{MD}, \mathrm{PhD}$ \\ ${ }^{1}$ Department of Ophthalmology, Teikyo University School of Medicine, Tokyo, Japan \\ ${ }^{2}$ Department of Ophthalmology, Tokyo Women's Medical University Medical Center East, Tokyo, Japan \\ ${ }^{3}$ Department of Ophthalmology, University of Tokyo Graduate School of Medicine, Tokyo, Japan \\ ${ }^{4}$ Department of Ophthalmology, Hachioji Medical Center, Tokyo Medical University, Tokyo, Japan \\ ORCID: \\ Tatsuya Mimura: https://orcid.org/0000-0001-5593-5338
}

J Ophthalmic Vis Res 2020; 15 (2): 270-272

Dear Editor,

Conjunctivochalasis is a common ocular condition characterized by excess conjunctival folds and is associated with aging. ${ }^{[1-4]}$ We previously demonstrated that the progression of conjunctivochalasis after sclerocorneal tunnel incisions was associated with the axial length of the eyeball and placement of conjunctival sutures. ${ }^{[4]}$ Conjunctival chemosis occurs more frequently in cases of sclerocorneal tunnel incisions than in cases of corneal incisions. ${ }^{[5]}$ This suggests that a clear corneal incision may induce less postoperative conjunctival inflammation compared to a transconjunctival sclerocorneal incision. Therefore, we evaluated the influence of clear corneal incision in cataract surgery on the severity of conjunctivochalasis based on a previously reported grading scale..$^{[1]}$

This study was conducted in accordance with the tenets of the Declaration of Helsinki and

\section{Correspondence to:}

Tatsuya Mimura, MD, PhD. Department of Ophthalmology, Teikyo University School of Medicine, 2-11-1 Kaga, Itabashi-ku, Tokyo 173-8605, Japan.

E-mail: mimurat-tky@umin.ac.jp

Received: 07-06-2019 Accepted: 04-09-2019

\section{Access this article online}

Website:

https://knepublishing.com/index.php/JOVR

DOI:

10.18502/jovr.v15i2.6749 was approved by the Institutional Review Board. The inclusion criteria were: age $>40$ years; nuclear cataract grades II-IV based on the Emery-Little classification; and uncomplicated phacoemulsification surgery. A total of 83 patients (83 eyes) who underwent mini-incision $(2.4 \mathrm{~mm}$ ) phacoemulsification for corneal wound were enrolled, including 43 men and 40 women aged $72.3 \pm 9.8$ years (mean \pm standard deviation), with an age range of $42-88$ years.

The severity of conjunctivochalasis was assessed using a modified grading system as previously described. ${ }^{[1,2]}$ Cataract surgery was performed via a clear corneal incision created with a $2.4 \mathrm{~mm}$ knife at the superior or temporal position. We evaluated the severity of conjunctivochalasis after one week and one, three, and six months postoperatively. The total conjunctivochalasis score significantly increased after postoperative month $3(P=0.0017)$ but decreased after postoperative month $6(P=0.5806)$ [Figure 1]. Progression of conjunctivochalasis was defined as $\geq 2$-point increase in the total conjunctivochalasis score. Table 1 shows the results of the multivariate analysis using a stepwise

This is an open access journal, and articles are distributed under the terms of the Creative Commons Attribution-NonCommercial-ShareAlike 4.0 License, which allows others to remix, tweak, and build upon the work non-commercially, as long as appropriate credit is given and the new creations are licensed under the identical terms.

How to cite this article: Mimura $T$, lida M, Noma $H$, Kamei $Y$, Kondo A, Yoshida M, Oguri M, Tanaka Y, Mizota A. . J Ophthalmic Vis Res 2020;15:270-272. 


\begin{tabular}{lccr}
\hline \multicolumn{2}{l}{ Table 1. Multivariate analysis of factors influencing progression of conjunctivochalasis ( $\mathrm{n}=83)}$. \\
\hline Variable & OR & $(95 \%$ Cl) & P-value \\
\hline Age (years) & 1.01 & $(1.00-1.02)$ & 0.0660 \\
Gender (male) & 1.21 & $(0.97-1.51)$ & 0.0895 \\
Hypertension & 1.11 & $(0.90-1.37)$ & 0.3341 \\
Diabetes & 1.00 & $(0.79-1.27)$ & 0.9964 \\
Hyperlipidemia & 0.94 & $(0.66-1.34)$ & 0.7317 \\
Coronary heart disease & 1.14 & $(0.87-1.50)$ & 0.3435 \\
Refraction (diopters) & 0.99 & $(0.96-1.02)$ & 0.3293 \\
Anterior chamber depth & 0.89 & $(0.68-1.17)$ & 0.3972 \\
Lens thickness & 1.00 & $(0.85-1.19)$ & 0.9589 \\
Axial Length (mm) & 1.01 & $(0.93-1.10)$ & 0.8196 \\
Corneal incision (superior / temporal) & 1.06 & $(0.84-1.34)$ & 0.6190 \\
Baseline total conjunctivochalasis score & 0.90 & $(0.85-0.95)$ & 0.0001
\end{tabular}

OR, odds ratio; $\mathrm{Cl}$, confidence interval

The total conjunctivochalasis score was calculated as the sum of the scores for the temporal, central, and nasal regions (0-9).



Figure 1. Mean postoperative total conjunctivochalasis score at each time point.

selection of factors associated with progression of conjunctivochalasis. The baseline total conjunctivochalasis score was significantly associated with the progression of conjunctivochalasis (odds ratio $=0.90, P=0.0001$ ).

In this study, the postoperative total conjunctivochalasis score increased after postoperative week 1 but subsequently returned to the preoperative level. Additionally, the preoperative severity of conjunctivochalasis was an independent determinant of the postoperative progression of conjunctivochalasis. These results suggest that a clear corneal incision did not change the severity of conjunctivochalasis.

The severity of conjunctivochalasis showed a significant increase after the first postoperative week, which was probably due to postoperative conjunctival inflammation, as there was a subsequent return to baseline. We found that the axial length influences the severity of conjunctivochalasis. ${ }^{[3]}$ Our previous study had also demonstrated that the axial length was independently associated with the grade of conjunctivochalasis after adjustment for age. ${ }^{[3]}$

In conclusion, our results showed no significant change in the severity of conjunctivochalasis after 24 postoperative weeks despite a transient early increase. This article complements our previous study on the progression of conjunctivochalasis after sclerocorneal tunnel incisions.

\section{Financial Support and Sponsorship}

The Ministry of Education, Culture, Sports, Science and Technology of Japan provided financial support in the form of a Grant-in-Aid for Scientific Research (16K11332). The sponsor had no role in the design or conduct of this research. 


\section{Conflicts of Interest}

There are no conflicts of interest.

\section{REFERENCES}

1. Meller D, Tseng SC. Conjunctivochalasis: literature review and possible pathophysiology. Surv Ophthalmol 1998;43:225-232.

2. Mimura T, Yamagami S, Usui T, Funatsu $H$, Mimura $Y$, Noma $\mathrm{H}$, et al. Changes of conjunctivochalasis with age in a hospital-based study. Am J Ophthalmol 2009;147:171177.

3. Mimura T, Yamagami S, Kamei Y, Goto M, Matsubara M. Influence of axial length on conjunctivochalasis. Cornea 2013;32:1126-1130.

4. Mimura $T$, lida $M$, Oshima R, Noma $H$, Kamei $Y$, Goto $\mathrm{M}$, et al. Changes of conjunctivochalasis after cataract surgery via a superior transconjunctival sclerocorneal incision. Int Ophthalmol 2017;37:691-700.

5. Sugai S, Yoshitomi F, Oshika T. Transconjunctival singleplane sclerocorneal incisions versus clear corneal incisions in cataract surgery. I Cataract Refract Surg 2010;36:1503-1507. 